\title{
Choices and Tradeoffs: Decreasing Costs or Improving Hospital Mortality Rates
}

Ann E. Tourangeau

\section{The Issue}

There is wide variation in risk- and case mix-adjusted hospital 30-day mortality rates for acutely ill medical patients across Ontario hospitals, and mean risk-adjusted hospital mortality rates for these patients increased between 1999 and 2003 (Table 1). In 1998/99, this rate for acutely ill medical patients (those with acute myocardial infarction [AMI], stroke, pneumonia, and septicemia or blood poisoning) was $15 \%$ and in $2002 / 03$, the mean mortality rate for this same patient population was $17.4 \%$. The span or variation of mortality rates in 2002/03 was $9.9 \%$ to $28.3 \%$. Known reasons for variation in mortality rates include patients' own characteristics and their associated risk factors as well as structures and processes of hospital care (quality). But once patients' characteristics and their associated risks have been accounted for, as in the rates identified in Table 1, why does wide variation in rates persist across hospitals? It is reasonable to suspect that much of the remaining variation is related to the quality of hospital care - the structures and processes of care. Because nurses provide most of the ongoing care to hospitalized patients, it is reasonable to also suspect that hospital nursing structures and processes are related to quality indicators such as mortality rates.

\section{Previous Canadian Research}

Previous research by the Institute for Clinical Evaluative Sciences (ICES) has found that three hospital variables explained $32 \%$ of variance in Ontario teaching and community hospital risk-adjusted 30-day mortality rates for acutely ill medical patients throughout 1998-1999. Lower hospital mortality rates were associated with a higher proportion of registered nurses (RNs) in the nursing staff mix (as opposed to licensed/registered practical nurses or unlicensed assis- tive personnel), more years of nurse experience on the current medical unit and more missed shifts by nurses (increased sick time). A similar study in Alberta found that a nursing staff mix richer in RNs was associated with lower 30-day mortality rates and that hospitals with a higher proportion of baccalaureate or university-educated nurses caring for acutely ill medical patients had lower mortality rates.

\section{The Current Study}

In the most recent study, ICES and University of Toronto scientists investigated the association between hospital quality (structures and processes of care) and risk-adjusted 30-day mortality rates for the same population of patients throughout 2002-2003. They surveyed nurses working in medical and
Table 1. Hospital 30-Day Mortality Rates for Acutely III Medical Patients in Ontario Hospitals*

\begin{tabular}{|l|c|c|}
\hline Fiscal Year & Mean & Range \\
\hline $1998-1999$ & $15 \%$ & $10.5-21.5 \%$ \\
\hline $2002-2003$ & $17.4 \%$ & $9.9-28.3 \%$ \\
\hline
\end{tabular}

${ }^{*} n=75$ teaching and community hospitals. Adapted from Tourangeau et al. (2007).
Table 2. Description of Study Patients by Diagnosis Group

\begin{tabular}{|l|r|r|r|c|c|}
\hline & \multicolumn{1}{|c|}{ AMI } & Stroke & Pneumonia & Septicemia & All Patients \\
\hline Number of cases & 19,475 & 11,160 & 13,433 & 2,925 & 46,993 \\
\hline Mean age (yr) & 69 & 74 & 72 & 70 & 71 \\
\hline$\%$ male & 63 & 50 & 49 & 48 & 55 \\
\hline Crude mortality rate & 13 & 21 & 16 & 31 & 16.9 \\
\hline
\end{tabular}

$\mathrm{AMI}=$ acute myocardial infarction . Adapted from Tourangeau et al. (2007). 
combined medical-surgical units in 75 Ontario teaching and community hospitals between February and May 2003. The scientists linked these nurse survey data with data on 46,993 patients admitted to hospital with AMT, stroke, pneumonia and septicemia between April 1, 2002, and March 31, 2003, in order to determine nursing-related elements that impact 30-day mortality. Table 2 describes patients included in this study by diagnosis group.

\section{Some hospitals may choose to ignore the evidence and continue to substitute RNs with less expensive nursing personnel.}

Eight variables explained $45 \%$ of variation in Ontario hospital mortality rates. Lower 30-day mortality rates were associated with (1) a higher proportion of RNs in the nursing staff mix, (2) a higher proportion of university-educated nurses, (3) higher nurse-reported adequacy of staffing and other resources, (4) increased use of care maps/protocols to guide patient care, (5) lower total dose (number) of nursing personnel, (6) higher nurse-reported quality of care, (7) lower nurse-reported manager ability and support and (8) higher nurse burn-out. Specifically, a $10 \%$ increase in proportion of RNs providing care to acutely ill medical patients was associated with six fewer deaths per 1,000 discharged patients. A $10 \%$ increase in university-educated nurses caring for these patients was associated with an additional nine fewer deaths per 1,000 discharged patients. And, a $10 \%$ increase in nurse-reported adequacy of staffing and other resources was associated with 17 fewer deaths per 1,000 discharged patients.

\section{Discussion}

These findings point to at least three ways that hospital quality can be improved to lower mortality rates. Yet risk-adjusted mortality rates are increasing rather than holding steady or decreasing for this patient group in Ontario hospitals. Why is this so? Why have hospitals decreased their proportions of RNs in their nurse staffing mix (75\% in 1999 and 66\% in 2003)? There are a number of possible explanations.

First, hospitals are not routinely held accountable for quality outcomes such as mortality rates to the same extent that they are held accountable for financial outcomes. Because it has been difficult for hospitals to calculate their own risk-adjusted rates, this outcome often remains invisible. Consequently, some hospitals may choose to ignore the evidence and continue to substitute RNs with less expensive nursing personnel. Others might argue that we are in the midst of an acute and persisting shortage of RNs. This shortage is related to baby boomer retirement, inadequate replacement of retirees with newly educated nurses (recruitment), increasing demand for RNs (e.g., for an increasingly aging population with more healthcare needs) and, more recently, difficulty retaining new nurses in hospital work environments that are less than optimal.

If hospitals have goals of minimizing unnecessary patient death for their acute medical patients, they should maximize the proportion of RNs providing direct care, aggressively seek to hire and retain university-educated nurses and make a significant investment in the development, use and systematic updating of care maps or protocols to guide patient progress throughout hospitalization. HQ

\section{Bibliography}

Estabrooks, C.A., W.K. Midodzi, G.G. Cummings, K.L. Ricker and P. Giovannetti. 2005. "The Impact of Hospital Nursing Characteristics on 30-Day Mortality.” Nursing Research 54(2): 74-84.

Silber, J.H., P.R. Rosenbaum and R.N. Ross. 1995. "Comparing the Contributions of Groups of Predictors: Which Outcomes Vary with Hospital rather than Patient Characteristics?" Journal of the American Statistical Association 90(429): 7-18.

Tourangeau, A.E. 2003. "Modeling the Determinants of Mortality for Hospitalized Patients." International Nursing Perspectives 3(1): 37-48.

Tourangeau, A.E. 2005. "A Theoretical Model of the Determinants of Mortality." Advances in Nursing Science 28(1): 58-69.

Tourangeau, A.E. 2006. "Taux de mortalité à 30 jours ajustés sur le diagnostic pour les patients hopitalisés en soins de courte durée dans les hôspitaux de l'Ontario, Canada." Risques et Qualite 3(1): 25-31.

Tourangeau, A.E., A.L. Coghlan, J. Shamian and S. Evans. 2005. "Registered Nurse and Registered Practical Nurse Evaluations of Their Hospital Practice Environments and Their Responses to These Environments." Canadian Journal of Nursing Leadership 18(4): 54-69.

Tourangeau, A.E. and L.A. Cranley. 2006. "Nurse Intention to Remain Employed: Understanding and Strengthening Determinants.” Journal of Advanced Nursing 55(4): 497-509.

Tourangeau, A.E., L.A. Cranley and L. Jeffs. 2006. "Impact of Nursing on Hospital Patient Mortality: A Focused Review and Related Policy Implications." Quality and Safety in Health Care 15(1): 4-8.

Tourangeau, A.E., D.M. Doran, L. McGillis Hall, L. O’Brien Pallas, D. Pringle, J.V. Tu and L.A. Cranley. 2007. "Impact of Hospital Nursing Care on 30-Day Mortality for Acute Medical Patients." Journal of Advanced Nursing 57(1): 32-44.

Tourangeau, A.E., P. Giovannetti, J. Tu and M. Wood. 2002. "NursingRelated Determinants of 30-Day Mortality for Hospitalized Patients." Canadian Journal of Nursing Research 33(4): 71-88.

Tourangeau, A.E. and J.V. Tu. 2003. "Developing Risk-Adjusted 30Day Hospital Mortality Rates." Research in Nursing and Health 26(6): 483-96.

\section{About the Author}

Ann E. Tourangeau, RN, PhD, is an adjunct scientist with the Institute for Clinical Evaluative Sciences and an associate professor at the Lawrence S. Bloomberg Faculty of Nursing at the University of Toronto, Toronto, Ontario. She holds a Career Scientist award (2003-2008) from the Ontario Ministry of Health and Long-Term Care. For more information, contact Ann Tourangeau at ann.tourangeau@utoronto.ca. 


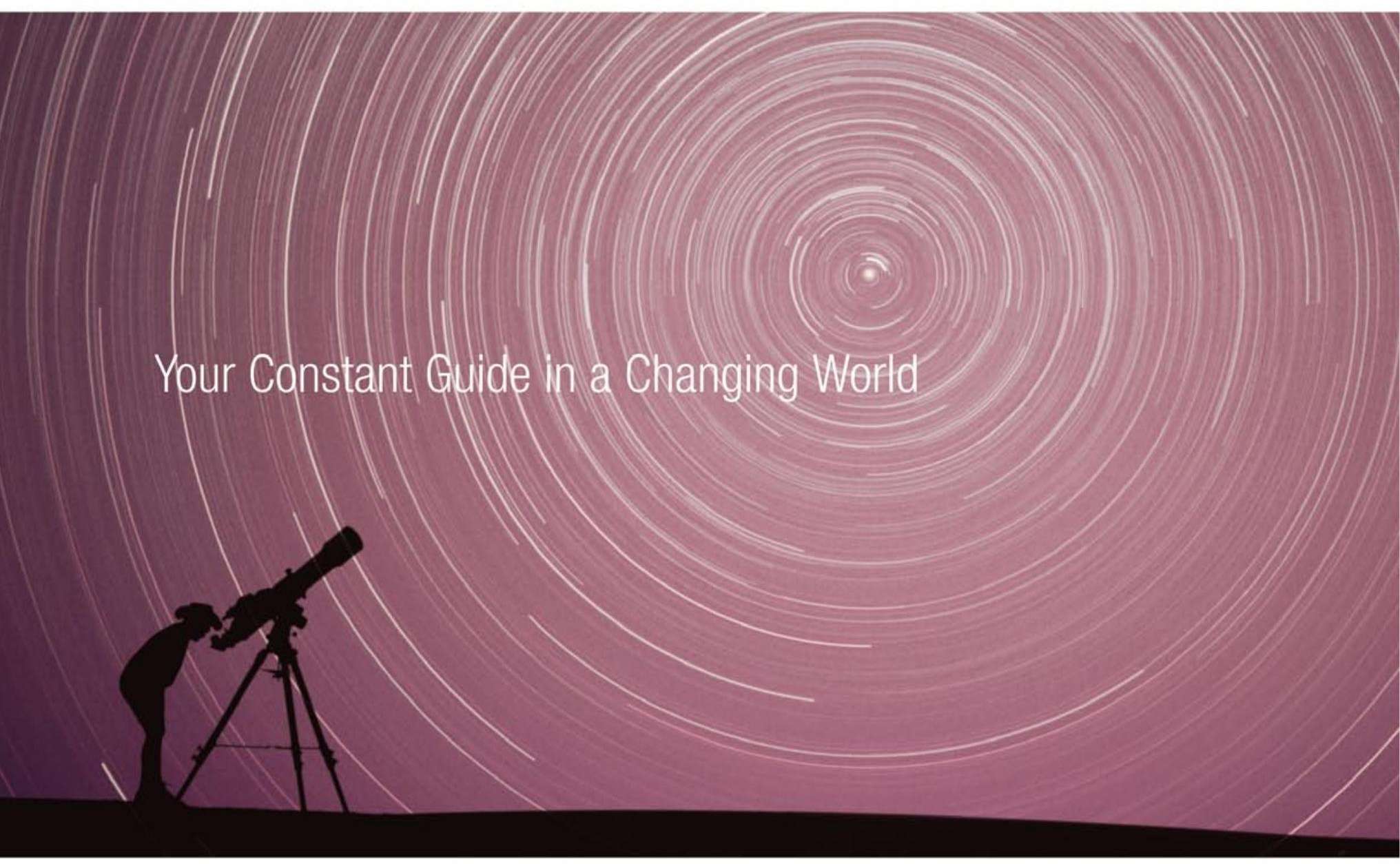

\section{Look to 3M to Keep Your Health Care Organization on Course}

In today's environment of changing compliance regulations and evolving industry standards, it's easy to lose your bearings. Fortunately, nobody knows the territory like $3 \mathrm{M}$ Health Information Systems. For more than 15 years, Canadian health care organizations have utilized $3 \mathrm{M}$ and SoftMed products to:

* Dramatically increase productivity, virtually eliminating scanning backlog

* Significantly reduce transcription costs and turnaround time

* Reduce redundant data collection and enhance data integrity

No matter how the health care world changes, with $3 \mathrm{M}$ Health Information System's recent merger with SoftMed, you can count on the experts at $3 \mathrm{M}$ to help you prepare, adapt and thrive.

www.3M.com/ca/HIS

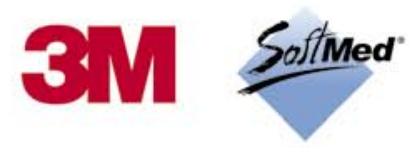

